\title{
The immature spine: growth and idiopathic scoliosis
}

\author{
Alain Dimeglio ${ }^{1}$, Federico Canavese ${ }^{2}$ \\ ${ }^{1}$ Pediatric Orthopedic Department, Clinique St. Roch, Montpellier, France; ${ }^{2}$ Pediatric Surgery Department, University Hospital Estaing, Clermont- \\ Ferrand, France \\ Contributions: (I) Conception and design: All authors; (II) Administrative support: F Canavese; (III) Provision of study materials or patients: All \\ authors; (IV) Collection and assembly of data: All authors; (V) Data analysis and interpretation: All authors; (VI) Manuscript writing: All authors; (VII) \\ Final approval of manuscript: All authors. \\ Correspondence to: Alain Dimeglio, MD. Professor of Pediatric Surgery, Pediatric Orthopedic Department, Clinique St. Roch, 560 Avenue du Colonel \\ Pavelet, 34000 Montpellier, France. Email: alaindimeglio@wanadoo.fr.
}

\begin{abstract}
Untreated progressive scoliosis can have negative effects on the growing spine as asymmetrical forces will act on the growth plates of the vertebral column (>130 growth plates). Spinal growth can be considered as a mixture of hierarchy, synchronization, and harmony: the slightest error can lead to a complex malformation; it is also a very dynamic process although it does not progress linearly: periods of acceleration are followed by periods of deceleration. Remaining growth is a determining factor for the worsening of idiopathic scoliosis (IS): the younger is the child, the higher is the risk of progression, and the more severe will be the disease. After birth, growth of the spine is not linear, and three periods can be identified: (I) between birth and age 5 years; (II) between age 5 and 10 years of age; (III) between age 10 and skeletal maturity. Spine and thoracic cage growth are correlated, although their growth is not synchronous. Timely control of the spinal deformity and its correction are mandatory to restore-as soon as possible-the harmony and the hierarchy of growth between the different growth plates. If action is delayed, the abnormal growth and the subsequent anatomical modifications will lead to a progressive, evolutive, and irreversible clinical picture. This article aims to provide a comprehensive review of how spinal deformities can affect the normal spine and thoracic cage growth.
\end{abstract}

Keywords: Idiopathic scoliosis (IS); thoracic growth; thoracic volume; spine growth

Submitted Nov 11, 2019. Accepted for publication Nov 22, 2019.

doi: 10.21037/atm.2019.11.134

View this article at: http://dx.doi.org/10.21037/atm.2019.11.134

\section{Introduction}

Volumetric growth and ossification of the spine represent an exceptionally long and slow process that starts around the third month of intrauterine life and does not end until the second decade of life. Idiopathic scoliosis (IS) can have negative effects on the growing spine as asymmetrical forces will act on the growth plates of the vertebral column. For this reason, IS is an evolutive growth plate disorder as, overall, more than 130 growth plates working in perfect synchronization, are involved in spinal growth (1).

Spinal growth can be considered as a mixture of hierarchy, synchronization, and harmony: the slightest error can lead to a complex malformation; it is also a very dynamic process although it does not progress linearly: periods of acceleration are followed by periods of deceleration (1-4).

As a result, standing height is not the best parameter to assess spinal growth because it does not directly measure the spinal column. Therefore, the best parameter to monitor and to evaluate spinal growth is the sitting height. In addition, spinal growth should always be considered in conjunction with the thoracic cage as the two entities are closely related.

Remaining growth is a determining factor for the worsening of IS: the younger is the child, the higher is the risk of progression, and the more severe will be the disease. Puberty is a turning point in children with IS as the pubertal growth spurt increases the risk of progression $(3,4)$. 
Timely control of the spinal deformity, as well as its correction, is necessary to restore-as soon as possible - the harmony and the hierarchy of growth between the different growth plates. If action is delayed, the abnormal growth and the subsequent anatomical modifications will lead to a progressive, evolutive, and irreversible clinical picture.

\section{Infantile IS: from birth to age $\mathbf{5}$ years}

The first five years of life are characterized by a significant increase in the sitting height. During this period (birth to age 5 years), the sitting height will increase of about $27-30 \mathrm{~cm}$; in particular, sitting height is about $35 \mathrm{~cm}$ at birth and about $62 \mathrm{~cm}$ at age 5 years. In particular, the first two years of life are characterized by the significant growth of the spine as sitting height will get 22 out of $35 \mathrm{~cm}(63 \%)(2,5)$.

It is important to note that the gain in spinal height (in $\mathrm{cm}$ ) occurring during the first 5 years of life is the same as the gain between age 5 years and skeletal maturity.

Growth of the spine cannot be dissociated from the thoracic cage growth, as these two entities are closely interrelated. The growth of the thoracic cage can be considered as the "fourth dimension of the spine". In particular, the thoracic perimeter is a valuable indicator of the thoracic cage growth (2).

The thorax has a perimeter of about $32 \mathrm{~cm}$ at birth. During the first five years of life (birth to age 5 years), the thoracic perimeter will increase of about $24 \mathrm{~cm}$. At birth, thoracic perimeter makes up $98 \%$ of the sitting height, and it has reached about $36 \%$ of its final size. By age 5 years, the thoracic perimeter will reach $63 \%$ of its final size $(1,2,5)$.

Severe infantile scoliosis leads to progressive deformity of the thoracic cage, and it can negatively influence both the thoracic cage and cardio-respiratory system growth. Over time, an orthopedic disorder (scoliosis = crooked spine $=$ bone disorder) becomes a systemic pediatric disease characterized by severe spine distortion and cardiopulmonary impairment. This disease is characterized by a cascade of growth disorders that amplify each other, like a "domino effect": an abnormal growth (spine) leads to another abnormal growth (thorax), which leads to an additional abnormal growth (cardio-pulmonary system). The goal of treatment is to break this vicious circle by restoring thoracic motion as well as enough space for the lungs and heart (parasol effect). In this respect, an offensive strategy is essential to protect lung development and growth. Canavese et al. have experimentally proven that an early spinal arthrodesis can have negative effects on the development of the thoracic cage and the lungs (6-10). Karol et al. have confirmed the negative consequences of early spine fusion in children with 1an early-onset spinal deformity in a clinical setting (11). A spinal arthrodesis of the thoracic spine performed early in life can have has negative repercussions on various organic chest components, heart, lungs, respiratory muscles, nervous system, and endocrine system. There is an interaction between the organic components of the spine, the thoracic cage, the intra-thoracic, and some extra-thoracic organs. Fusion is the cause of respiratory insufficiency and adds to the spinal deformity of the loss of pulmonary function (6-11).

At birth, the thoracic volume has reached only $6 \%$ and $30 \%$ of its definitive size at birth and age 5 years, respectively. During this period (birth to age 5 years), the segment T1-S1 will increase of about $10 \mathrm{~cm}, 2 / 3$ at the level of the thoracic spine, and $1 / 3$ at the level of the lumbar spine. During this period, the spine is still poorly ossified (30\% to $40 \%$ ), and it is mostly cartilaginous, thus vulnerable to the changes induced by a progressive spinal deformity.

The T1-S1 segment measures about $18 \mathrm{~cm}$ at birth and reaches about $45 \mathrm{~cm}$ at skeletal maturity. A thoracic spine (T1-T12 segment) of at least 18 to $22 \mathrm{~cm}$ is necessary to avoid respiratory compromise as it corresponds to about $70 \%$ of expected T1-T12 length $(1,2,5,12)$.

Moreover, careful weight assessment is an important part of the orthopedic evaluation. At birth, weight is about $3 \mathrm{~kg}$, and it reaches about $20 \mathrm{~kg}$ by age 5 years $(32 \%$ of final weight). Furthermore, most children with progressive infantile scoliosis are shown to exhibit a deficit in weight $(1,2,5,12,13)$.

For all these reasons, progressive infantile deformities are much more than a simple orthopedic disorder and do need a multidisciplinary approach. At skeletal maturity, the goal is to have a vital capacity of at least $50 \%$, a weight of at least $40 \mathrm{~kg}$, and a thoracic spine length of at least 18 to $22 \mathrm{~cm}(2,11-13)$.

\section{Juvenile IS: from 5 to 10 years of age}

After 5 years of age, and until age 10 years, the annual growth rate of the spine slows down, and it is a deceleration period. During this period, all indicators of growth do not progress at the same speed, although they are all interdependent and somehow synchronized.

Between the ages of 5 and 10, the annual growth rate of their spine slows down. In particular, the gain is sitting 
height is about $2.5 \mathrm{~cm} /$ year, while the T1-S1 spine segment will gain about 1.1 to $1.2 \mathrm{~cm} /$ year. Overall from age 5 years to skeletal maturity, the sitting height will increase of about $30 \mathrm{~cm}$, and during this period, the annual weight increase is of about $2.5 \mathrm{~kg} /$ year $(1,2,5)$.

The thoracic perimeter reaches about $56 \mathrm{~cm}$ at age 5 years and is about $66 \mathrm{~cm}$ by age 10 years (annual growth rate of about $2 \mathrm{~cm} /$ year); this increase is, however, lower compared to the first five years of life (13).

A progressive spinal deformity leading to distortion of the thoracic cage can compromise lung growth because up to $85 \%$ of alveoli develop after birth. In particular, from birth to age 7 years, there is an increase in the number of alveoli (alveolar multiplication), while after that age, there is an increase in the alveolar size and the overall number of alveoli does not change anymore (alveolar growth). The thoracic volume will reach $50 \%$ of its definitive size by age 10 years (30\% by age 5 years), while alveolar multiplication will end at around age 7 years (the normal number of alveoli increase from 20 million at birth to 300 million at age 7 years). For such reason, alteration of spinal and thoracic growth during the period of alveolar multiplication can potentially compromise both alveolar and lung growth $(2,12,13)$.

Thoracic cage shape and function play an essential role in lung growth. As the curve progresses, not only the growth of the spine is reduced, but the size and function of the thoracic cage are affected as well. Therefore, the crucial period for both spine and thoracic cage growth occurs during the first 5 to 7 years of life, and it coincides with lung development. Gollogly et al. reported that lung parenchyma volume is a function of age. Lung parenchyma volume is about $400 \mathrm{ccs}$ at birth, $900 \mathrm{ccs}$ at age 5 years, 1,500 ccs at age 10 years, and about 4,000 ccs at skeletal maturity (14).

By age 10 years, $60 \%$ of the spine is ossified (ossification was $30 \%$ at age 5 years) $(1,2)$.

Bracing and serial casting are two of the most recommended treatment options for patients with juvenile scoliosis; the goal of treatment is to slow down the progression of the deformity in order to buy time up to the beginning of puberty when spine fusion can potentially be performed. It is, therefore, important to take advantage of this quiescent period of spinal growth (15-17). Charles et al. have shown that if curve magnitude reaches $30^{\circ}$ at the beginning of puberty, the surgical risk is very close to $100 \%$; on the other hand, the surgical risk is about $20 \%$ if the curve magnitude at the beginning of puberty is $20^{\circ}(18)$.

In children with progressive deformity, there is a decrease of longitudinal growth and a loss of the normal proportionality of trunk growth, and the loss of sitting height highlights this phenomenon. The loss of sitting height is related to the severity of the deformity.

\section{Adolescent IS: the pubertal growth spurt}

Puberty is a turning point in children with IS as the pubertal growth spurt increases the risk of deformity progression. Duval-Beaupère et al. have showed that during a pubertal growth spurt, as a rule of thumb, scoliosis will worsen substantially in most cases (19-22).

Perfect knowledge of the distinct stages of puberty is essential to supply a rationale, and well balanced, treatment strategy.

Chronological age is a poor indicator of remaining growth and pubertal growth spurt; as a rule of thumb, bone age should be preferred (23-26).

Puberty starts at 11 years of bone age in girls and 13 years of bone age in boys. The beginning of puberty is characterized by an acceleration of growth, not only at the level of the spine. A dramatic increase in stature, a significant change in the proportions of the upper and lower body segments, a modification of the overall morphology of the body, and the development of the secondary sexual characteristics are typical of this period.

During the pubertal growth spurt, growth is far more noticeable in the trunk than in the lower limbs; in particular, during puberty, $2 / 3$ of growth is at the level of the trunk (sitting height) and $1 / 3$ at the level of the lower extremity (sub-ischial length). At skeletal maturity, the thoracic spine (T1-T12 segment) represents about $30 \%$ of sitting height, and one single thoracic vertebra and its disc represent about $2.5 \%$ of sitting height. Similarly, the lumbar spine (L1-L5 segment) is about $19 \%$ of sitting height, and one single lumbar vertebra and its disc do stand for about $3.5 \%$ of the sitting height $(1,2)$.

During puberty, standing height increases by 1.4 to $1.5 \mathrm{~cm} /$ year, and a new period of acceleration of growth can be observed. It is for this very specific reason that most spinal deformities tend to worsen substantially during puberty $(2-4,27,28)$.

At the beginning of puberty, boys have $13 \%$ of remaining growth, which represents about $22.5 \mathrm{~cm}(12.5 \mathrm{~cm}$ in sitting height and $10 \mathrm{~cm}$ in sub-ischial length). Similarly, girls have $12 \%$ of remaining growth, which represents about $20.5 \mathrm{~cm}$ (11.5 cm in sitting height and $9 \mathrm{~cm}$ in sub-ischial length).

The peak growth velocity occurs between 13 and 15 years of bone age in boys and between 11 and 13 years 
of bone age in girls. After this period, there is a substantial decrease in the annual growth rate, with the lower limbs stopping to grow rapidly. At the end of the peak height velocity, the remaining growth is about $5.5 \mathrm{~cm}(4 \mathrm{~cm}$ in the sitting height and $1.5 \mathrm{~cm}$ in the lower limbs).

Secondary sexual characteristics develop throughout puberty. The first physical sign of puberty in girls is breast budding; the first sign of puberty in boys is the testicular growth. It is important to note that secondary sexual characteristics generally develop in harmony with bone age.

By plotting the gain in sitting and standing height every 6 months, a clear picture of the pubertal growth spurt can be developed (pubertal diagram). In particular, two very distinct periods can be identified: (I) the acceleration phase, between 11 to 13 and 13 to 15 years of bone age in girls and boys, respectively; this is the so-called "ascending side of the pubertal growth spurt"; (II) the deceleration phase, between 13 to 15 and from 15 to 17 years of bone age in girls and boys, respectively; this is the so-called "descending side of the puberty" characterized by a significant deceleration of growth. During the ascending side, the gain in sitting height is approximately $8.5 \mathrm{~m}$ in boys and $7.7 \mathrm{~cm}$ in girls; on the other hand, the gain is about $5 \mathrm{~cm}$ for boys and $4.5 \mathrm{~cm}$ for girls during the descending side $(3,4,18,24)$.

The spine surgeon must be aware of the following radiographic parameters:

* Risser sign appears during the descending phase; Risser 1 appears at 13.5 years and 15.5 years of bone age in girls and boys, respectively. Risser 1 usually corresponds to the onset of menarchis in girls;

* Triradiate cartilage closes at 12 years of bone age in girls, and at 14 years of bone age in boys, the closure occurs during the ascending side;

* Elbow closure (13 and 15 years of bone age in girls and boys, respectively) and fusion of the distal phalanx of the hand split the pubertal growth spurt into two parts: ascending and descending side;

* The peak height velocity is not a point in the curve as it takes place during the first 2 years of puberty (ascending side);

* During puberty, the peak of growth results from a combination of three smaller peaks (micro-peaks): the first peak involves the lower limbs, the second peak involves the trunk, and the third peak involves the thorax.

Whatever the population or the ethnicity, the chronology of puberty is always the same: an acceleration phase (ascending side) followed by a deceleration phase (descending side).

The pubertal diagram is a reference, and the following basic question show is asked when dealing with children with progressive scoliosis: where is the child located on the pubertal diagram? What about the remaining growth?

It is also essential to consider the bone age of the patients, as only $50 \%$ of the population has a bone age in concordance with the chronological age. There are many options to assess bone age. In our opinion, the simplified olecranon method should be preferred during the ascending phase of puberty as it is more precise than the Greulich and Pyle atlas. On the descending phase, the Risser sign is helpful although not precise enough when complex decisions must be taken; during this period, methods involving the hand are more detailed although a good correlation has been proved between hand and iliac crest radiographs $(19,22,23,25,27-29)$.

Risser 0 makes up $2 / 3$ of puberty; as a result, the Risser 0 period must be divided into two parts: (I) Risser 0-Triradiate Cartilage open (11 to 12 and 13 to 14 years of bone age in girls and boys, respectively) and (II) Risser 0-Triradiate Cartilage closed (12 to 13 and 14 to 15 years of bone age in girls and boys, respectively).

When plotting all these data on the pubertal diagram, four distinct zones can be identified: two in the ascending side and 2 in the descending side $(2,18,23,26)$ :

* Zone 1: Risser 0, Triradiate Cartilage open (ascending side);

* Zone 2: Risser 0, Triradiate Cartilage closed (ascending side);

* Zone 3: Risser 1-2, Greater Trochanter open (descending side);

* Zone 4: Risser 3-5, Greater Trochanter closed (descending side).

It is essential to evaluate the behavior of the curve during the ascending side of the pubertal diagram, when the risk of progression is higher (critical period) to evaluate the potential for progression of spinal curvature (aggressivity).

Despite all useful information bone age can provide, it must always be balanced with secondary sexual characteristics, annual growth velocity, and, in girls, the onset of menarchis. Sometimes Risser 5 (complete ossification of the iliac apophysis) never appears, and it is, therefore, difficult to decide whether a specific treatment can be stopped or not: clinical signs are important! If no more sitting height increase is identified, treatment can be stopped. Usually, 2 years after the onset of menarchis, the trunk stops growing $(1,2,5)$. 


\section{The crankshaft phenomenon}

There is only one way to control a scoliotic curve: to neutralize all growth plates involved in the deformity at once! This fundamental concept is at the heart of the crankshaft phenomenon.

The crankshaft phenomenon is omnipresent. Any time a scoliotic curve us treated by posterior instrumented fusion, the patient becomes exposed to the crankshaft phenomenon $(29,30)$. In patients with infantile scoliosis, the risk of crankshaft phenomenon is the highest, and any hardware can prevent it from occurring. During the pubertal growth spurt, the risk persists. Sanders et al. have proved that the risk of crankshaft phenomenon, is lower when the triradiate cartilage is closed (31). However, triradiate cartilage closes one year after the onset of puberty, and at that point remaining sitting height is about 8 to $9 \mathrm{~cm}$ in both boys and girls $(1,2,5,18,24,26)$.

The ascending side of puberty, between Risser 1 and Risser 5, is characterized by a significant development of the thoracic cage, which can aggravate further the deformity of the spine. Full screws constructs can reduce the risk of a crankshaft, although the risk cannot completely be eliminated.

The crankshaft phenomenon should not only be merely considered as worsening of the Cobb angle as it carries several other negative effects: aggravation of the spinal imbalance, deterioration of the thoracic deformity, and aggravation of the deformity above and below the instrumented segments $(29,30)$.

\section{The 'scoliotic risk'}

When dealing with a patient with IS, the basic question is, "what is the scoliotic risk of the patient?" As a rule of thumb, the younger the child, the higher the scoliotic risk because of the higher amount of remaining growth.

Scoliosis of $20^{\circ}$ has not the same risk of deterioration at 5,10 , or 15 years of age! Several papers have been published on the surgical risk of scoliosis, and by merging all published data, a simple approximation can potentially be used in daily practice $(18,24)$ :

The scoliotic risk for a $20^{\circ}$ curve should be multiplied by 5 at 5 years of age $(20 \times 5=100 \%$ risk of progression), by 4 at 10 years of age $(20 \times 4=80 \%$ risk of progression), by 3 at the beginning of puberty $(20 \times 3=60 \%$ risk of progression) and by 2 at the peak of pubertal growth spurt (Risser I; $20 \times 2=40 \%$ risk of progression).
At Risser 2, the risk of deterioration of scoliosis of a $20^{\circ}$ curve is about $20 \%$, although more severe deformities $\left(30^{\circ}\right.$ to $40^{\circ}$ ) carry a higher risk of progression $(30 \%)(18,24)$.

Therefore, a clear distinction must be made between curves of measuring $10^{\circ}, 20^{\circ}$, or $30^{\circ}$, when the patient is on the descending side of puberty, as the strategy of management depends on the topography of the curve. In particular, the lumbar deformity should never be underestimated as untreated progressive curves carry the poorest outcome during adulthood.

A curve increasing by $10^{\circ}$ during the ascending side of puberty (first 2 years of puberty) carries an elevated risk of requiring surgical treatment.

\section{What will be the final deficit of the trunk after posterior spine instrumented fusion?}

This recurrent question is raised when dealing with severe, evolutive curves in skeletally immature patients.

The deficit in trunk height induced by spine fusion can be easily reassessed by (I) evaluating the sitting height; (II) evaluating the remaining sitting height; (III) considering each single thoracic vertebra will cause a $2.5 \%$ deficit is sitting height and that each single lumbar vertebral will cause a deficit of $3.5 \%$ of sitting height. These values have to be adjusted to the age of the patient! Some authors have suggested a $0.07 \mathrm{~mm} /$ vertebra/year of remaining growth $(1,2,5)$.

When dealing with severe and very severe scoliosis at the beginning of puberty, despite the risk of crankshaft phenomenon, surgical treatment by posterior instrumented fusion is a rationale choice because the correction of the deformity will compensate the overall loss of sitting height.

\section{Conclusions}

Spinal and thoracic cage growth follows strict rules. It is necessary to analyze all growth parameters to fully understand the deficits induced by progressive scoliosis over a growing organism. It must also be kept in mind that the thoracic cage is frequently part of the problem, as deeply linked to the spine. There is a reciprocal interaction between the spine, thoracic cage, and cardio-respiratory system. Progressive spinal deformities and early spinal arthrodesis can alter spinal and thoracic cage growth by altering both shape function. Correction and control of the spinal deformity are needed to restore-as soon as possible-a harmonious growth pattern. If action is delayed, or not taken, the abnormal growth and the later anatomical 
modifications of both spine and the thoracic cage will lead to a progressive, evolutive and irreversible clinical picture.

The following key-points are important and should always be considered:

* The growing spine is a complex phenomenon as more than 130 growth plates contribute to it;

* Repeating clinical measurements allow to identify progressive deformities and to treat by anticipation;

* Sitting height is more precise than standing height;

* Bone age is more precise than chronological age;

* Ask yourself: (I) What is the sitting height? (II) What about remaining sitting height? (III) What is bone age? (IV) Is the patient on the ascending or descending side of puberty?

- Most spinal deformities arise within the T1-S1 segment (strategic segment);

* The T1-S1 segment makes up about $49 \%$ of the sitting height at skeletal maturity;

* The T1-T12 segment (thoracic spine) represents about $30 \%$ of the sitting height (at maturity);

* The L1-L5 segment (lumbar spine) represents about $19 \%$ of the sitting height (at skeletal maturity);

* Spine and thoracic care growth are deeply interrelated;

* About $50 \%$ of trunk growth occurs during the first 5 years of life;

* The thoracic cage distortion associated with progressive scoliosis is part of the problem; in this respect, the thoracic cage should be considered as the "fourth dimension of the spine";

* As the spinal deformity progresses, by a "domino effect", not only spinal growth is affected but the size and shape of the thoracic cage are modified as well;

* The crankshaft phenomenon is at the heart of pediatric spinal pathology;

* The timing of spinal arthrodesis should be decided after taking into account all growth parameters.

\section{Acknowledgments}

None.

\section{Footnote}

Conflicts of Interest: The authors have no conflicts of interest to declare.

Ethical Statement: The authors are accountable for all aspects of the work in ensuring that questions related to the accuracy or integrity of any part of the work are appropriately investigated and resolved.

\section{References}

1. Dimeglio A, Bonnel F. Le rachis en croissance. Paris, France: Springer Verlag 1990.

2. Dimeglio A, Canavese F. The growing spine: how spinal deformities influence the normal spine and thoracic cage growth. Eur Spine J 2012;21:64-70.

3. DiMeglio A, Canavese F, Charles YP. Adolescent Idiopathic Scoliosis: when and how much? J Pediatr Orthop 2011;31:S28-36.

4. Dimeglio A, Canavese F. Progression or not progression? How to deal with adolescent idiopathic scoliosis during puberty. J Child Orthop 2013;7:43-9.

5. Dimeglio A. Growth of the spine before age 5 years. J Pediatr Orthop B 1993;1:102-7.

6. Canavese F, Dimeglio A, Volpatti D, et al. Dorsal arthrodesis of thoracic spine and effects on thorax growth in prepubertal New Zealand white rabbits. Spine 2007;32:E443-50.

7. Canavese F, Dimeglio A, D'Amato C, et al. Dorsal arthrodesis in prepubertal New Zealand White rabbits followed to skeletal maturity: effect on thoracic dimensions, spine growth, and neural elements. Indian J Orthop 2010;44:14-22.

8. Canavese F, Dimeglio A, Granier M, et al. Arthrodesis of the first six dorsal vertebrae in prepubertal New Zealand White rabbits and thoracic growth to skeletal maturity: the role of the "Rib-Vertebral-Sternal complex." Minerva Ortop Traumatol 2007;58:369-78.

9. Canavese F, Dimeglio A, Granier M, et al. Influence de l'arthrodèse vertébrale sélective T1-T6 Sur la croissance thoracique: étude expérimentale chez des lapins New Zealand White prépubertaires. Rev Chir Orthop Reparatrice Appar Mot 2008;94:490-7.

10. Canavese F, Dimeglio A, Stebel M, et al. Thoracic Cage Plasticity in Prepubertal New Zealand White Rabbits Submitted to T1-T12 Dorsal Arthrodesis: Computed Tomography Evaluation, Echocardiographic Assessment and Cardio-pulmonary Measurements. Eur Spine J 2013;22:1101-12.

11. Karol LA, Johston C, Mladenov K, et al. Pulmonary function following early thoracic fusion in non neuromuscular scoliosis. J Bone Joint Surg Am 2008;90:1272-81.

12. Canavese F, Dimeglio A, Bonnel F, et al. Thoracic cage 
volume and dimension assessment by optoelectronic molding in normal children and adolescents during growth. Surg Radiol Anat 2019;41:287-96.

13. Canavese F, Dimeglio A. Normal and abnormal spine and thoracic cage development. World J Orthop 2013;4:167-74.

14. Gollogly S, Smith JT, White SK, et al. The volume of lung parenchyma as a function of age: a review of 1050 normal CT scans of the chest with three-dimensional volumetric reconstruction of the pulmonary system. Spine 2004;29:2061-6.

15. Canavese F, Kaelin A. Adolescent idiopathic scoliosis: indications and efficacy of non-operative treatment. Indian J Orthop 2011;45:7-14.

16. Canavese F, Samba A, Dimeglio A, et al. Serial elongationderotation-flexion casting for children with early-onset scoliosis. World J Orthop 2015;6:935-43.

17. Canavese F, Botnari A, Dimeglio A, et al. Serial elongation, derotation and flexion (EDF) casting under general anesthesia and neuromuscular blocking drugs improve outcome in patients with juvenile scoliosis: preliminary results. Eur Spine J 2016;25:487-94.

18. Charles YP, Daures JP, De Rosa V, et al. Progression risk of juvenile idiopathic scoliosis during pubertal growth. Spine 2006;31:1933-42.

19. Duval-Beaupère G, Dubousset J, Queneau P. Pour une théorie unique de l'évolution des scolioses. Presse Med 1970;78:1141.

20. Duval-Beaupère G, Combes J. Segments supérieur et inférieur au cours de la croissance physiologique des filles: étude longiᄀtudinale de la croissance de 54 filles. Arch Fr Pediatr 1971;28:1057.

21. Duval-Beaupère G. Les repères de maturation dans la surveillance des scolioses. Rev Chir Orthop 1970;56:59.

22. Duval-Beaupère G. Croissance résiduelle de la taille et des

Cite this article as: Dimeglio A, Canavese F. The immature spine: growth and idiopathic scoliosis. Ann Transl Med 2020;8(2):22. doi: 10.21037/atm.2019.11.134 segments après la première menstruation chez la fille. Rev Chir Orthop 1976;62:501.

23. Canavese F, Charles YP, Dimeglio A. Skeletal age assessment from elbow radiographs. Review of the literature. Chir Organi Mov 2008;92:1-6.

24. Charles YP, Diméglio A, Canavese F, et al. Skeletal age assessment from the olecranon for idiopathic scoliosis at Risser grade 0. J Bone Joint Surg Am 2007;89:2737-44.

25. Diméglio A, Charles YP, Daures JP, et al. Accuracy of the Sauvegrain method in determining skeletal age during puberty. J Bone Joint Surg Am 2005;87:1689-96.

26. Canavese F, Charles YP, Dimeglio A, et al. A comparison of the simplified olecranon and digital methods of assessment of skeletal maturity during the pubertal growth spurt. Bone Joint J 2014;96-B:1556-60.

27. Charles YP, Marcoul A, Schaeffer M, et al. Threedimensional and volumetric thoracic growth in children with moderate idiopathic scoliosis compared with normal. J Pediatr Orthop B 2017;26:227-32.

28. Charles YP, Canavese F, Dimeglio A. Curve progression risk in a mixed series of braced and non-braced patients with idiopathic scoliosis related to skeletal maturity assessment on the olecranon. J Pediatr Orthop B 2017;26:240-4.

29. Dubousset J. Recidive d'une scoliose lombaire et d'un basin oblique après fusion precoce: le phénomène du Villebrequin. Proceeding Group Etude de la Scoliose; Paris, France 1973.

30. Dubousset J, Herring JA, Shufflebarger HL. The crankshaft phenomenon. J Pediatr Orthop 1989;9:541.

31. Sanders JO, Khoury JG, Kishan S, et al. Predicting scoliosis progression from skeletal maturity: a simplified classification during adolescence. J Bone Joint Surg Am 2008;90:540-53. 